\title{
Impurity effective mass in superfluid ${ }^{4} \mathrm{He}$
}

\author{
I.O.Vakarchuk, V.V.Babin \\ Ivan Franko Lviv State University, Department for Theoretical Physics, \\ 12 Drahomanov St., UA-290005 Lviv, Ukraine \\ Received June 9, 1998
}

The system of bose liquid + impurity is considered. The energy spectrum, as well as effective mass of impurity is calculated. For the case of an ${ }^{3} \mathrm{He}$ atom in superfluid ${ }^{4} \mathrm{He}$ numerical calculations are performed.

Key words: superfluid helium, impurity, effective mass

PACS: $67.60 .-g, 05.30 .-d$

\section{Introduction}

A couple of the most interesting questions are to be mentioned within the problem of determining the impurity energy spectrum in superfluid ${ }^{4} \mathrm{He}$, namely, the calculation of the impurity effective mass $M^{*}$ and revealing the spectrum character in the region of wave vectors corresponding to the reverse interatom distance. Within the framework of the thermodynamic approach the effective mass of ${ }^{3} \mathrm{He}$ was originally determined on the basis of experimental data for heat capacity and the spin diffusion coefficient by J.Bardeen, G.Baym, D.Pines [2]. They found the value $M^{*} / M=2.34$ for the impurity effective mass and the isolated atom $M$ mass ratio in the case of the liquid ${ }^{4} \mathrm{He}$ equilibrium density. A microscopic theory of the energy spectrum for ${ }^{3} \mathrm{He}$ impurities in superfluid ${ }^{4} \mathrm{He}$ was independently developed in $[1,3-6,11]$. Considering the case of a single ${ }^{3} \mathrm{He}$ atom, T.B.Devison and E.Feenberg [3] made use of the Brillouin-Wigner perturbation theory and calculated the impurity spectrum branch, having chosen as zero approximation wave functions the wave functions of pure ${ }^{4} \mathrm{He}$ and an isolated ${ }^{3} \mathrm{He}$ atom. The authors determined the energy of replacement, the effective mass and a relative change of liquid bulk caused by the replacement of ${ }^{4} \mathrm{He}$ with ${ }^{3} \mathrm{He}$. In order to obtain the numerical value of $M^{*}$, a liquid ${ }^{4} \mathrm{He}$ structure factor calculated theoretically by different authors was used. For example, using the data of [7], the value $M^{*} / M=1.81$ is obtained. The same problems were considered by Slyusarev and Strzhemechny [4]; they used a trial variation function of an impure atom and the BrillouinWigner perturbation theory with a long wave estimate for the matrix elements of 
"impurity" and "impurity-phonon". An explicit expression for the impurity effective mass via the ${ }^{4} \mathrm{He}$ structure factor, as well as the numerical value $M^{*} / M=2.4$ on the basis of the experimentally measured structure factor were given in that work, too. The authors noted an essential non-square behaviour of the spectrum in the wave vector region near $3 \AA^{-1}$. A similar expression for $M^{*}$ was obtained by Woo Tan and Massey [5]; however, by using the theoretical structure factor they obtained $M^{*} / M=1.85$ to be closer to the value found in [3]. The same authors in [6] improved this result in $M^{*} / M=2.37$ by the evaluation of higher corrections to the effective mass of the interaction "impurity - phonon". In [8], a variational wave function suitable only for the calculation of the effective mass is chosen. With the help of this function, which takes into account the backflow arising from driving the ${ }^{3} \mathrm{He}$ atom in the form proposed by Feynman and Cohen for pure ${ }^{4} \mathrm{He}[9]$, the value $M^{*} / M=1.7$ is obtained. We would like to mention work [10] as well, in which the spectrum of the impurity and its dampings were investigated by means of the dynamic structure factor for small values of the wave vector $M^{*} / M=2.35$, as well as in the region of the roton minimum ${ }^{4} \mathrm{He}$. A "one-parameter" trial wave function without the application of the perturbation theory of Brillouin-Wigner is used in [11] for the calculation of the impurity branch of the spectrum. For the effective mass two first corrections are explicitly calculated, each of which contains one sum over a wave vector more than the previous one. Using experimental data for the structure factor, in the case of the ${ }^{3} \mathrm{He}$ atom the value $M^{*} / M=1.73$ is obtained.

In the present work the calculation of the impurity spectrum will be carried out on the basis of the polaron-type Hamiltonian where liquid helium + impurity system is simulated in the approximation of one sum over a wave vector. We propose to pass in the Hamiltonian to other variables so that impurity coordinates be dropped out, and further to develop the perturbation theory not for the interaction "liquid - impurity", but for an additional "anharmonic" term which results from such a transformation of independent variables. In the zero approximation of the approach the result for the spectrum of the impurity of the second order of the perturbation theory for the potential of the interaction "liquid - impurity" is reconstituted, but without the assumption of the smallness of the "liquid - impurity" interaction. Numerical estimates of the effective mass of the ${ }^{3} \mathrm{He}$ atom in superfluid ${ }^{4} \mathrm{He}$ on the basis of experimental values of the structural factor for liquid ${ }^{4} \mathrm{He}$ are also made.

\section{Notations}

The object of our study is a model of the system "superfluid ${ }^{4} \mathrm{He}+$ impurity", based on the following Hamiltonian:

$$
H=\frac{P^{2}}{2 M}+H_{\mathrm{He}^{4}}+H_{\mathrm{int}} .
$$

The first term in (1) represents a kinetic energy of the impurity atom (practically, it is the ${ }^{3} \mathrm{He}$ atom), the mass of which we have denoted as $M$. We take the Hamil- 
tonian of superfluid helium in the approximation of noninteracting elementary perturbations

$$
H_{\mathrm{He}^{4}}=E_{0}+\sum_{\boldsymbol{q} \neq 0} E_{q} b_{\boldsymbol{q}}^{\dagger} b_{\boldsymbol{q}}
$$

with the Bogolubov spectrum $E_{q}$ and energy of the ground state $E_{0}$ [12] :

$$
\begin{aligned}
& E_{0}=\frac{N(N-1)}{2 V} \nu_{0}-\frac{1}{4} \sum_{\boldsymbol{q} \neq 0} \frac{\hbar^{2} q^{2}}{2 m}\left(\alpha_{q}-1\right)^{2}, \\
& E_{q}=\frac{\hbar^{2} q^{2}}{2 m} \alpha_{q}, \quad \alpha_{q}=\sqrt{1+\frac{2 N}{V} \nu_{q} / \frac{\hbar^{2} q^{2}}{2 m}},
\end{aligned}
$$

where letters $N$ and $V$ denote the number of particles of the liquid and the volume of the system, respectively, $m$ is the mass of atom ${ }^{4} \mathrm{He}$ and $\nu_{q}$ is the Fourie-image of the pair potential of the inter-particle interaction in superfluid ${ }^{4} \mathrm{He}$. The operators of the creation-anihilation of elementary excitations $b_{\boldsymbol{q}}^{\dagger}, b_{\boldsymbol{q}}$ satisfy the commutative relations of Bose:

$$
\left[b_{\boldsymbol{q}}, b_{\boldsymbol{k}}^{\dagger}\right]=\delta(\boldsymbol{q}-\boldsymbol{k}) .
$$

The part of the Hamiltonian (1) which corresponds to the interaction between a liquid and impurity, is expressed in the following form:

$$
H_{\mathrm{int}}=\frac{N}{V} w_{0}+\frac{\sqrt{N}}{V} \sum_{\boldsymbol{q} \neq 0} \frac{w_{q}}{\sqrt{\alpha}_{q}} e^{i \boldsymbol{q} \boldsymbol{R}}\left(b_{-\boldsymbol{q}}^{\dagger}+b_{\boldsymbol{q}}\right) .
$$

Here $\boldsymbol{R}$ is a coordinate of the impurity, $w_{q}$ is the Fourie-image of the potential of the interaction between the ${ }^{4} \mathrm{He}$ atom and the impurity atom.

\section{Calculation of the impurity spectrum}

In order to treat the problem it is convenient to pass from (1) to the unitary equivalent Hamiltonian $H_{*}$

$$
H_{*}=U H U^{\dagger}
$$

with the help of the transformation

$$
U=\exp \left(i \sum_{\boldsymbol{q} \neq 0}(\boldsymbol{q} \boldsymbol{R}) b_{\boldsymbol{q}}^{\dagger} b_{\boldsymbol{q}}\right), \quad U^{\dagger}=U^{-1}
$$

$U$ means the "translation" of the ${ }^{4}$ He atoms radius-vectors $\boldsymbol{r}_{1}, \ldots, \boldsymbol{r}_{N}$ by $(-\boldsymbol{R})$, that is a passage from variables $\left(\boldsymbol{r}_{1}, \ldots, \boldsymbol{r}_{N}, \boldsymbol{R}\right)$ to $\left(\boldsymbol{r}_{1}-\boldsymbol{R}, \ldots, \boldsymbol{r}_{N}-\boldsymbol{R}, \boldsymbol{R}\right)$. The explicit expression for $H_{*}$ is as follows:

$H_{*}=E_{0}^{B}+\frac{N}{V} w_{0}+\frac{1}{2 M}\left(\boldsymbol{P}-\sum_{\boldsymbol{q} \neq 0} \hbar \boldsymbol{q} b_{\boldsymbol{q}}^{\dagger} b_{\boldsymbol{q}}\right)^{2}+\sum_{\boldsymbol{q} \neq 0} E_{q} b_{\boldsymbol{q}}^{\dagger} b_{\boldsymbol{q}}+\frac{\sqrt{N}}{V} \sum_{\boldsymbol{q} \neq 0} \frac{w_{q}}{\sqrt{\alpha}_{q}}\left(b_{\boldsymbol{q}}^{\dagger}+b_{\boldsymbol{q}}\right)$. 
Transformation (3) enables us to omit the impurity coordinates, but, instead of that, results in the emergence of anharmonic terms.

The operator of impulse $\boldsymbol{P}$ of the impurity now can be considered as a c-number and one can identify the spectrum of the impurity by the energy of the ground state which corresponds to $H_{*}$.

The Hamiltonian $H_{*}$, as well as input $H$, cannot be diagonalized exactly, therefore, for further calculations we use the theory of perturbations. It is natural next to divide $H_{*}$ into the problem which supposes an exact solution:

$$
H_{*}^{0}=\frac{N}{V} w_{0}+E_{0}+\frac{\boldsymbol{P}^{2}}{2 M}+\sum_{\boldsymbol{q} \neq 0}\left\{\left(E_{q}+\frac{\hbar^{2} q^{2}}{2 M}-\frac{\hbar}{M} \boldsymbol{q} \boldsymbol{P}\right) b_{\boldsymbol{q}}^{\dagger} b_{\boldsymbol{q}}+\frac{\sqrt{N}}{V} \frac{w_{q}}{\sqrt{\alpha}_{q}}\left(b_{\boldsymbol{q}}^{\dagger}+b_{\boldsymbol{q}}\right)\right\}
$$

and into the perturbation

$$
H_{*}^{\mathrm{int}}=\frac{\hbar^{2}}{2 M} \sum_{\boldsymbol{q}_{1} \neq 0} \sum_{\boldsymbol{q}_{2} \neq 0}\left(\boldsymbol{q}_{1} \boldsymbol{q}_{2}\right) b_{\boldsymbol{q}_{1}}^{\dagger} b_{\boldsymbol{q}_{2}}^{\dagger} b_{\boldsymbol{q}_{1}} b_{\boldsymbol{q}_{2}}
$$

(a normal ordering of the operators of the creation-anihilation is suggested). But, by using an arbitrariness of this separation, we introduce additional parameter $\phi(\boldsymbol{q})$, with the help of which we try to improve the outcome of the perturbation theory approach to the exact solution. In other words, let us assume

$$
H_{*}=\widetilde{H}_{*}^{0}+\widetilde{H}_{*}^{\text {int }}
$$

where

$$
\begin{aligned}
\widetilde{H}_{*}^{0} & =H_{*}^{0}+\sum_{\boldsymbol{q} \neq 0} \phi(\boldsymbol{q}) b_{\boldsymbol{q}}^{\dagger} b_{\boldsymbol{q}}, \\
\widetilde{H}_{*}^{\text {int }} & =H_{*}^{\text {int }}-\sum_{\boldsymbol{q} \neq 0} \phi(\boldsymbol{q}) b_{\boldsymbol{q}}^{\dagger} b_{\boldsymbol{q}} .
\end{aligned}
$$

In support of such a step it is also possible to mention the argument that no small parameter of the natural origin exists in the input Hamiltonian $H$.

We further develop the usual perturbation theory for the energy of the ground state $H_{*}$ :

$$
E=E^{(0)}+E^{(1)}+E^{(2)}+\ldots
$$

In the zero approximation the system under consideration is a set of harmonic oscillators in the inhomogeneous external field, therefore, without the assumption of the smallness of the liquid - impurity interaction, we obtain

$$
E^{(0)}=E_{0}+\frac{N}{V} w_{0}+\frac{\boldsymbol{P}^{2}}{2 M}-\frac{N}{V^{2}} \sum_{\boldsymbol{q} \neq 0} \frac{w_{q}^{2}}{\alpha_{q}\left(E_{q}+\frac{\hbar^{2} q^{2}}{2 M}-\frac{\hbar}{M}(\boldsymbol{q} \boldsymbol{P})+\phi(\boldsymbol{q})\right)}
$$


The first and the second corrections to $E^{(0)}$ have the following form:

$$
\begin{aligned}
& E^{(1)}=-\frac{N}{V^{2}} \sum_{\boldsymbol{q} \neq 0} \phi(\boldsymbol{q}) \frac{w_{q}^{2}}{\alpha_{q}\left(E_{q}+\frac{\hbar^{2} q^{2}}{2 M}-\frac{\hbar}{M}(\boldsymbol{q P})+\phi(\boldsymbol{q})\right)^{2}}+ \\
& +\frac{\hbar^{2}}{2 M}\left(\frac{N}{V^{2}} \sum_{\boldsymbol{q} \neq 0} \boldsymbol{q} \frac{w_{q}^{2}}{\alpha_{q}\left(E_{q}+\frac{\hbar^{2} q^{2}}{2 M}-\frac{\hbar}{M}(\boldsymbol{q} \boldsymbol{P})+\phi(\boldsymbol{q})\right)^{2}}\right)^{2} \\
& E^{(2)}=-\frac{N}{V^{2}} \sum_{\boldsymbol{q} \neq 0} \frac{w_{q}^{2}}{\alpha_{q}\left(E_{q}+\frac{\hbar^{2} q^{2}}{2 M}-\frac{\hbar}{M}(\boldsymbol{q P})+\phi(\boldsymbol{q})\right)^{3}} \times \\
& \times\left(\phi(\boldsymbol{q})-\frac{\hbar^{2}}{M} \frac{N}{V^{2}} \sum_{\boldsymbol{k} \neq 0}(\boldsymbol{q} \boldsymbol{k}) \frac{w_{k}^{2}}{\alpha_{k}\left(E_{k}+\frac{\hbar^{2} k^{2}}{2 M}-\frac{\hbar}{M}(\boldsymbol{k P P})+\phi(\boldsymbol{k})\right)^{2}}\right)^{2}+ \\
& +\frac{N^{2}}{V^{4}} \sum_{\boldsymbol{q} \neq 0} \frac{\left(\frac{\hbar^{2} q^{2}}{2 M}\right)^{2} w_{q}^{4}}{\alpha_{q}^{2}\left(E_{q}+\frac{\hbar^{2} q^{2}}{2 M}-\frac{\hbar}{M}(\boldsymbol{q P})+\phi(\boldsymbol{q})\right)^{5}}- \\
& -\frac{N^{2}}{V^{4}} \sum_{\boldsymbol{q}_{1} \neq 0} \sum_{\boldsymbol{q}_{2} \neq 0}\left(\frac{\hbar^{2}}{M}\left(\boldsymbol{q}_{1} \boldsymbol{q}_{2}\right)\right)^{2} \frac{w_{q_{1}}^{2}}{\alpha_{q_{1}}\left(E_{q_{1}}+\frac{\hbar^{2} q_{1}^{2}}{2 M}-\frac{\hbar}{M}\left(\boldsymbol{q}_{1} \boldsymbol{P}\right)+\phi\left(\boldsymbol{q}_{1}\right)\right)^{2}} \times \\
& \times \frac{w_{q_{2}}^{2}}{\alpha_{q_{2}}\left(E_{q_{2}}+\frac{\hbar^{2} q_{2}^{2}}{2 M}-\frac{\hbar}{M}\left(\boldsymbol{q}_{2} \boldsymbol{P}\right)+\phi\left(\boldsymbol{q}_{2}\right)\right)^{2}} \times \\
& \times \frac{1}{E_{q_{1}}+\frac{\hbar^{2} q_{1}^{2}}{2 M}-\frac{\hbar}{M}\left(\boldsymbol{q}_{1} \boldsymbol{P}\right)+\phi\left(\boldsymbol{q}_{1}\right)+E_{q_{2}}+\frac{\hbar^{2} q_{2}^{2}}{2 M}-\frac{\hbar}{M}\left(\boldsymbol{q}_{2} \boldsymbol{P}\right)+\phi\left(\boldsymbol{q}_{2}\right)} .
\end{aligned}
$$

In order to fix arbitrary function $\phi(\boldsymbol{q})$ it is possible to use a demand for the minimization of energy as a functional of $\phi(\boldsymbol{q})$, but we did not manage to solve the equations appearing in such an approach. We propose to require the vanishing of the first correction to $E^{(0)}$, that is to determine $\phi(\boldsymbol{q})$ from the condition $E^{(1)}=0$. Explicitly we have the equation :

$$
\begin{aligned}
& -\frac{N}{V^{2}} \sum_{\boldsymbol{q} \neq 0} \phi(\boldsymbol{q}) \frac{w_{q}^{2}}{\alpha_{q}\left(E_{q}+\frac{\hbar^{2} q^{2}}{2 M}-\frac{\hbar}{M}(\boldsymbol{q P})+\phi(\boldsymbol{q})\right)^{2}}+ \\
& +\frac{\hbar^{2}}{2 M}\left(\frac{N}{V^{2}} \sum_{\boldsymbol{q} \neq 0} \boldsymbol{q} \frac{w_{q}^{2}}{\alpha_{q}\left(E_{q}+\frac{\hbar^{2} q^{2}}{2 M}-\frac{\hbar}{M}(\boldsymbol{q P})+\phi(\boldsymbol{q})\right)^{2}}\right)^{2}=0 .
\end{aligned}
$$

The equation will be satisfied identically if one puts

$$
\phi(\boldsymbol{q})=\frac{\hbar}{M}(\boldsymbol{q} \boldsymbol{x})
$$

and imposes on the vector $\boldsymbol{x}$ the condition:

$$
\boldsymbol{x}=\frac{\hbar}{2} \frac{N}{V^{2}} \sum_{\boldsymbol{q} \neq 0} \boldsymbol{q} \frac{w_{q}^{2}}{\alpha_{q}\left(E_{q}+\frac{\hbar^{2} q^{2}}{2 M}-\frac{\hbar}{M}(\boldsymbol{q}, \boldsymbol{P}-x)\right)^{2}} .
$$


At $\boldsymbol{P}=0$ we hereof find $\boldsymbol{x}=0$. For small $\boldsymbol{P}$ let us assume that $\boldsymbol{x}=\lambda \boldsymbol{P}$, then, expanding the right-hand side (6) in the powers of $\boldsymbol{P}$ we obtain the following relation for the definition of $\lambda$ :

$$
\lambda=(1-\lambda) \frac{\sigma_{1}}{2}+o\left(P^{2}\right) .
$$

Here for the sake of simplicity we introduce

$$
\sigma_{1}=\frac{4}{3} \frac{N}{V^{2}} \sum_{\boldsymbol{q} \neq 0} \frac{w_{q}^{2} \frac{\hbar^{2} q^{2}}{2 M}}{\alpha_{q}\left(E_{q}+\frac{\hbar^{2} q^{2}}{2 M}\right)^{3}} .
$$

Hence, within the accuracy of the square and higher powers of the impulse of the impurity, we have

$$
\lambda=\frac{\sigma_{1}}{2+\sigma_{1}} .
$$

The usual theory of perturbations for $H_{*}^{i n t}$ corresponds to the value $\lambda=0$.

\section{Effective mass of the impurity}

The spectrum obtained above is in the region of small impulses and looks like

$$
E(P)=\varepsilon_{0}+\frac{P^{2}}{2 M^{*}},
$$

where $M^{*}$ is the so-called effective mass of the impurity atom, and the constant independent of the impulse equals

$$
\begin{aligned}
& \varepsilon_{0}=E_{0}+\frac{N}{V} w_{0}-\frac{N}{V^{2}} \sum_{\boldsymbol{q} \neq 0} \frac{w_{q}^{2}}{\alpha_{q}\left(E_{q}+\frac{\hbar^{2} q^{2}}{2 M}\right)}+ \\
& +\frac{N^{2}}{V^{4}} \sum_{\boldsymbol{q} \neq 0} \frac{\left(\frac{\hbar^{2} q^{2}}{2 M}\right)^{2} w_{q}^{4}}{\alpha_{q}^{2}\left(E_{q}+\frac{\hbar^{2} q^{2}}{2 M}\right)^{5}}-\frac{N^{2}}{V^{4}} \sum_{\boldsymbol{q}_{1} \neq 0} \sum_{\boldsymbol{q}_{2} \neq 0}\left(\frac{\hbar^{2}}{M}\left(\boldsymbol{q}_{1} \boldsymbol{q}_{2}\right)\right)^{2} \times \\
& \times \frac{w_{q_{1}}^{2}}{\alpha_{q_{1}}\left(E_{q_{1}}+\frac{\hbar^{2} q_{1}^{2}}{2 M}\right)^{2}} \frac{1}{\alpha_{q_{2}}\left(E_{q_{2}}+\frac{\hbar^{2} q_{2}^{2}}{2 M}\right)^{2}} \frac{1}{\left(E_{q_{1}}+\frac{\hbar^{2} q_{1}^{2}}{2 M}+E_{q_{2}}+\frac{\hbar^{2} q_{2}^{2}}{2 M}\right)} .
\end{aligned}
$$

The outcome of our calculations is the following expression for $M^{*}$ :

$$
\frac{M}{M^{*}}=1-\sigma_{1}+\sigma_{1}^{2}-\sigma_{1}^{3}-\sigma_{2}+2 \lambda\left(\sigma_{1}^{3}+\sigma_{2}\right)-\lambda^{2}\left(\sigma_{1}^{2}+\sigma_{1}^{3}+\sigma_{2}\right),
$$

where one more notation is introduced

$$
\sigma_{2}=\left(\frac{4}{3} \frac{N}{V^{2}}\right)^{2} \sum_{\boldsymbol{q}_{1} \neq 0} \sum_{\boldsymbol{q}_{2} \neq 0} \frac{\frac{\hbar^{2} q_{1}^{2}}{2 M} \frac{\hbar^{2} q_{2}^{2}}{2 M} w_{q_{1}}^{2} w_{q_{2}}^{2}}{\alpha_{q_{1}} \alpha_{q_{2}}\left(E_{q_{1}}+\frac{\hbar^{2} q_{1}^{2}}{2 M}\right)^{2}\left(E_{q_{2}}+\frac{\hbar^{2} q_{2}^{2}}{2 M}\right)^{2}} \times
$$




$$
\begin{aligned}
& \times \frac{1}{\left(E_{q_{1}}+\frac{\hbar^{2} q_{1}^{2}}{2 M}+E_{q_{2}}+\frac{\hbar^{2} q_{2}^{2}}{2 M}\right)}\left\{\frac { \hbar ^ { 2 } q _ { 1 } ^ { 2 } } { 2 M } \left[\frac{3}{\left(E_{q_{1}}+\frac{\hbar^{2} q_{1}^{2}}{2 M}\right)^{2}}+\right.\right. \\
& +\frac{2}{\left(E_{q_{1}}+\frac{\hbar^{2} q_{1}^{2}}{2 M}\right)\left(E_{q_{1}}+\frac{\hbar^{2} q_{1}^{2}}{2 M}+E_{q_{2}}+\frac{\hbar^{2} q_{2}^{2}}{2 M}+\right.}+ \\
& \left.+\frac{1}{\left(E_{q_{1}}+\frac{\hbar^{2} q_{1}^{2}}{2 M}+E_{q_{2}}+\frac{\hbar^{2} q_{2}^{2}}{2 M}\right)^{2}}\right]+\frac{\hbar^{2} q_{2}^{2}}{2 M}\left[\frac{3}{\left(E_{q_{2}}+\frac{\hbar^{2} q_{2}^{2}}{2 M}\right)^{2}}+\right. \\
& +\frac{1}{\left(E_{q_{2}}+\frac{\hbar^{2} q_{2}^{2}}{2 M}\right)\left(E_{q_{1}}+\frac{\hbar^{2} q_{1}^{2}}{2 M}+E_{q_{2}}+\frac{\hbar^{2} q_{2}^{2}}{2 M}+\right.}+ \\
& \left.\left.+\frac{1}{\left(E_{q_{1}}+\frac{\hbar^{2} q_{1}^{2}}{2 M}+E_{q_{2}}+\frac{\hbar^{2} q_{2}^{2}}{2 M}\right)^{2}}\right]\right\} .
\end{aligned}
$$

\section{Numerical calculations}

We provide numerical computations of the effective mass of the ${ }^{3} \mathrm{He}$ impurity in ${ }^{4} \mathrm{He}$ at $T=0 \mathrm{~K}$. We assume that the potentials of interatomic interactions ${ }^{3} \mathrm{He}-{ }^{4} \mathrm{He}$ and ${ }^{4} \mathrm{He}-{ }^{4} \mathrm{He}$ are identical, that is $\nu_{q}=w_{q}$, and further we express $\nu_{q}$ through the ${ }^{4} \mathrm{He}$ structure factor with the help of relations [1]:

$$
S_{q}=\frac{1}{\alpha_{q}}
$$

For the evaluations we have made use of measurements $S_{q}$ [13] up to the point $T=0 K$ by means of equalities [1]:

$$
S_{q}(T=0)=S_{q}(T) \tanh \left[\frac{E_{q}}{2 T}\right] .
$$

The density of ${ }^{4} \mathrm{He}$ is considered to be equal to $\rho^{4} \mathrm{He}=0.02185 \AA^{-3}$. The following values are obtained:

$$
\sigma_{1}=0.416, \quad \sigma_{2}=0.222 .
$$

And, accordingly, $M^{*} / M=1.82$. The usual theory of perturbations $(\lambda=0)$ gives $M^{*} / M=2.15$.

\section{References}

1. Bogolubov N.N. Izv.AN SSSR, ser. phys., II, 77, 1947 (in Russian).

2. Bardeen J., Baym G., Pines D. Phys. Rev., 156, 207, 1967. 
3. Davison T.B., Feenberg E. Phys. Rev., 178, 306, 1969.

4. Slyusarev V.A., Strzhemechny M.A. Ukr. Fiz. Zhurn., 14, 453, 1969 (in Russian).

5. Woo C-W., Tan H.-T., Massey W.E. Phys. Rev. Lett., 22, 278, 1969.

6. Woo C-W., Tan H.-T., Massey W.E. Phys. Rev., 185, 287, 1969.

7. Massey W.E., Woo C-W. Phys. Rev., 164, 256, 1967.

8. Owen J. C. Phys. Rev. B, 23, 5815, 1981.

9. Feynman R. P., Cohen M. Phys. Rev., 102, 1189, 1956.

10. Gotze W., Lucke M. Szprynger. Preprint Max-Planck-Institut für Physik und Astrophysik, München, 1979.

11. Vakarchuk I.O. The impurity energy spectrum in liquids. International school on the Physics of ionic solvation. Lviv, May 30 - June 5, 1983. Abstracts, Kiev, 1983, p. 31-32.

12. Bogolubov N.N., Zubarev D.N. ZhETPh, 28, 129, 1955 (in Russian).

13. Svensson E.C., Sears V.F., Woods A.D.B., Martel P. Phys. Rev. B, 21, 8, 1980.

\title{
Ефективна маса домішки в надплинному ${ }^{4} \mathrm{He}$
}

\author{
І.О.Вакарчук, В.В.Бабін
}

Львівський державний університет ім. І.Я.Франка, Кафедра теоретичної фізики, 290005 м. Львів, вул. Драгоманова, 12

Отримано 9 червня 1998 р.

Розглядається система “бозе-рідина + домішка". Розраховано енергетичний спектр домішки таїї ефективну масу. Проведено числові розрахунки для випадку атома ${ }^{3} \mathrm{He}$ в рідкому ${ }^{4} \mathrm{He}$.

Ключові слова: надплинний гелій, домішки, ефективна маса

PACS: $67.60 .-g, 05.30 .-d$ 